\title{
Characterizing spatio-temporal coupling of extreme ultraviolet ultrashort pulses from high harmonic generation
}

\author{
Adam S. Wyatt, Tobias Witting, Antoine Monmayrant and \\ Ian A. Walmsley \\ Clarendon Laboratory, University of Oxford, Parks Road, Oxford, OX1 3PU, UK \\ tel: +441865 282641, fax: +441865272400,a.wyatt1@physics.ox.ac.uk \\ Charles Haworth, Joseph S. Robinson, John W. G. Tisch \\ and Jonathon P. Marangos \\ Blackett Laboratory, Imperial College London, Prince Consort Road, London, \\ $S W 72 A Z$
}

\begin{abstract}
We demonstrate a tool for performing measurements of space-time coupling of ultrashort, extreme ultraviolet pulses from high harmonic generation which can be used to study propagation and phasematching effects during the generation process.

(C) 2011 Optical Society of America

OCIS codes: (320.7100) Ultrafast measurements; (020.4180) Multiphoton processes; (140.7240) UV, XUV, and X-ray lasers; (140.7090) Ultrafast lasers
\end{abstract}

Over the past few years, high harmonic generation (HHG) has become a popular method of generating ultrashort duration pulses (as low as several hundred attoseconds) in the extreme ultraviolet (XUV) spectral region. The drive for these sources have been greatly enhanced by the development of metrology and understanding of the HHG process. To date, current metrology has been directed towards measuring the spatial or temporal coherence, the spatial wavefront or the temporal duration of pulses generated via HHG [1].

In this contribution, we show a simple method to measure spatio-temporal coupling (STC) in XUV HHG. This gives the complete amplitude and phase correlations across the beam, which are important in verifying models of HHG. This method is particularly well suited for studying propagation effects (including phasematching) in the HHG source, which are of paramount importance. It also has potential applications in tomographic imaging of wavefunctions[2] and should allow one to perform pump-probe experiments in the XUV.
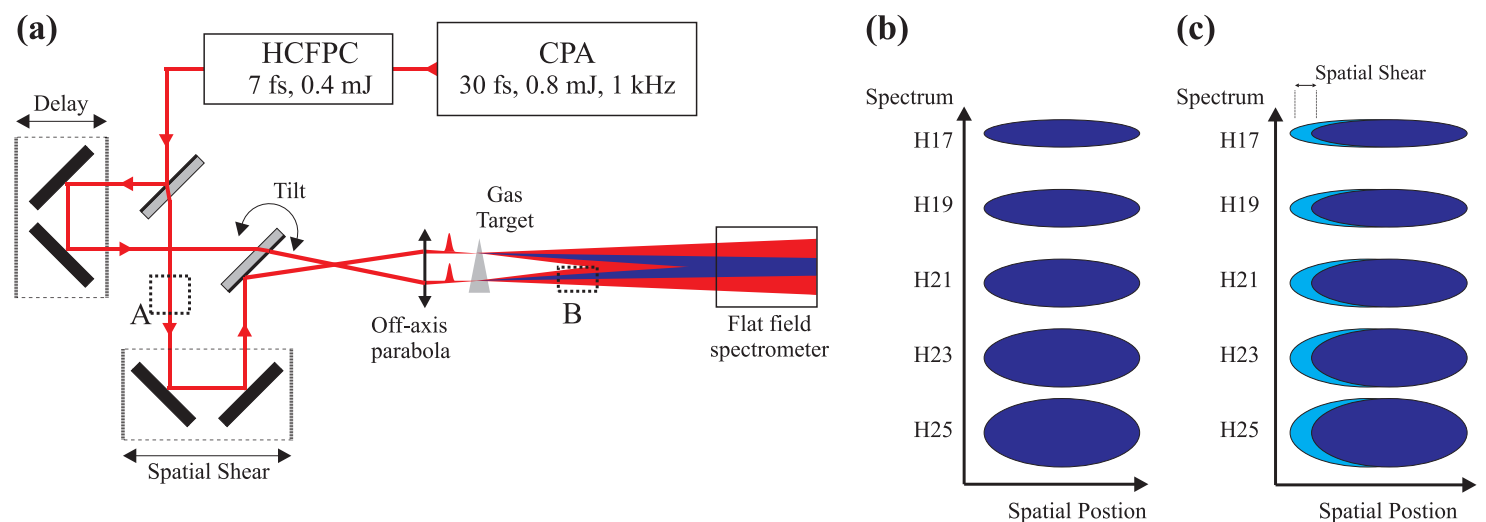

Fig. 1. (a) Schematic of experimental setup, (b) Calibration Trace - 2 pulses spatially overlap, (c) Spatial Shearing Interferometry - 2 pulses spatially separated.

To implement this method we compress a pulse from a Ti:S CPA system in a neon filled hollow-core fiber pulse compressor to produce 7 fs pulses at $1 \mathrm{kHz}$ repetition rate. Using a folded Mach-Zehnder interferometer, we generate two infrared (IR) pulses which are slightly spatially displaced and have a small tilt between them. Such a setup allows one to vary the tilt, spatial separation and time delay between the two pulses (figure 1). An off-axis $40 \mathrm{~cm}$ parabola is used to focus the two pulses into an argon gas target in such a way that the two focal regions are completely spatially separated to produce two independent coherent sources of high harmonics which then propagate so that they completely overlap spatially onto the MCP of a spatially 
resolving XUV spectrometer. The spectrometer used has no focusing in the spatial domain so that the spatial information of the harmonics are preserved.

The tilt between the two XUV pulses results in fringes in the spatial domain can be described by:

$I(\omega, x)=|E(\omega, x)|^{2}+|E(\omega, x-X)|^{2}+2|E(\omega, x)||E(\omega, x-X)| \cos \left\{\phi(\omega, x)-\phi(\omega, x-X)+\frac{2 \pi x}{\lambda} \sin (\delta \theta)\right\}$

where $\delta \theta$ is the propagation angle between the two beams and $X$ is the spatial shear. From this interference pattern, one can retrieve the relative phase and amplitude between the two high harmonics sources, in both the spatial and spectral domains.

As a first step, we have successfully generated two identical harmonics as shown in figure 2. Individual spectrum from each source are identical [figure 2 (a) and 2 (b)] and the relative phase extracted from the interferogram is perfectly flat. This can be seen intuitively in figure 2 (c) by the equally spaced straight fringes. The tilt on the fringes is only due to the k-vector being frequency dependant.

(a)

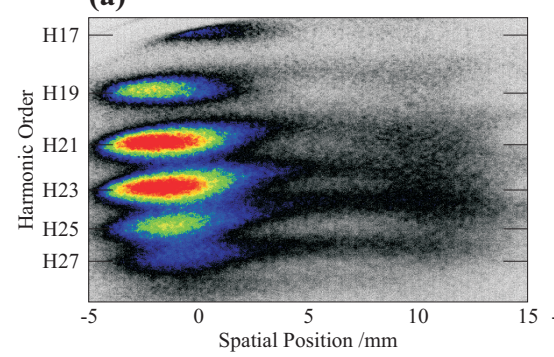

(b)

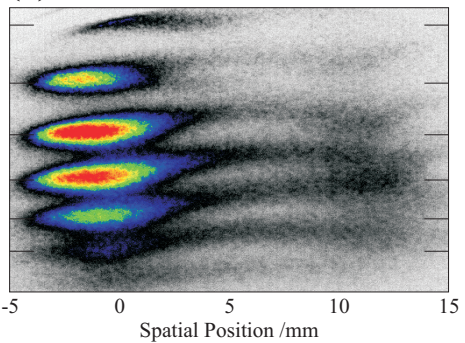

(c)

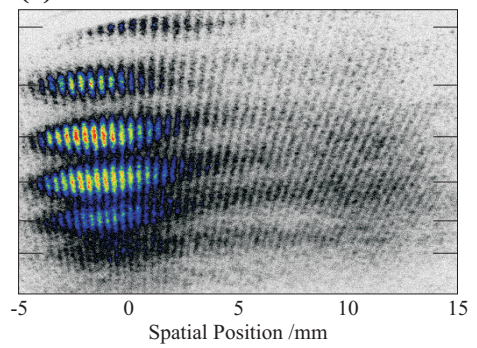

Fig. 2. (a) Harmonic spectrum as a function of spatial position for 1 arm of the interferometer, (b) harmonics spectrum for the other interferometer arm and (c) spatial interference pattern generated from both arms.

Although it is possible to deduce some information from the spectrum alone, full knowledge of the spatial phase as well as the spatial profile allows one to propagate the harmonics back to the gas medium where the spatio-temporal structure can be compared to simulations. To measure the spatial phase, and hence the pulse wavefront, a spatial shear is generated by translating one of the beams horizontally such that the two pulses are no longer completely spatially overlapped on the MCP. Any space-time coupling shows up as a modulation in the fringe pattern. This technique has proved useful in testing optical components using visible or infrared light. We believe that extending this technique to the XUV allows further studies of the generation process. For example, one could study the effect of seed beam properties (aperture, chirp rate, energy, focussing, ...) on harmonics generation, by altering the properties of one of the arms [represented by box $\mathrm{A}$ in figure 1 (a)] and record the evolution in phase and amplitude of the spatial and spectral profiles of the corresponding harmonics.

Providing two sources of attosecond XUV pulses also paves the way to reference based time-resolved spectroscopic measurements. As an example, it is possible to measure the spatio-temporal response of a sample placed in one of the XUV beams [represented by box B in figure 1 (a)]. Such a method can prove useful in designing new XUV optics, or for studying the scatter from a surface via a probe-probe experiment. Due to the interference of the two pulses, one also gains the advantage of homodyning, whereby the signal from a weak probe is enhanced by a much stronger reference beam.

In summary, we have demonstrated a simple approach to measuring the space-time coupling of ultrashort XUV pulses via the use of spatial shearing interferometry. The method is based on a well established technique used in the visible domain which is robust to noise and can also be measured single shot. We believe that such a method can prove valuable in further understanding propagation and phase-matching effects in high harmonic generation, as well as providing a tool for performing reference based time-resolved spectroscopy.

\section{References}

1. M. Hentschel, R. Kienberger, C. Spielmann, G. A. Reider, N. Milosevic, T. Brabec, P. Corkum, U. Heinzmann, M. Drescher, and F. Krausz, "Attosecond metrology," Nature 414(6863), 509-513 (2001). URL http://dx.doi.org/10.1038/35107000.

2. J. Itatani, J. Levesque, D. Zeidler, H. Niikura, H. Pepin, J. C. Kieffer, P. B. Corkum, and D. M. Villeneuve, "Tomographic imaging of molecular orbitals," Nature 432(7019), 867-871 (2004). URL http://dx.doi.org/10.1038/nature03183. 